\title{
Efficacy of reduced-fluence photodynamic therapy for serous retinal pigment epithelial detachment with choroidal hyperpermeability
}

This article was published in the following Dove Press journal:

Clinical Ophthalmology

29 October 2013

Number of times this article has been viewed

\section{Asako Shibata \\ Yasuhiro Ohkuma \\ Takaaki Hayashi \\ Hiroshi Tsuneoka \\ Department of Ophthalmology, The Jikei University School of Medicine, Tokyo, Japan}

Correspondence: Asako Shibata Department of Ophthalmology, The Jikei University School of Medicine, 3-25-8 Nishi-shimbashi, Minato-ku, Tokyo I05-846I, Japan

Tel +8I 33433 IIII (ext 358I)

Fax +8I 33433 |936

Email asako9o3@hotmail.co.jp
Background: Very few reports have addressed methods of treatment for idiopathic serous (IS) pigment epithelial detachment (PED).

Objective: The purpose of this report was to describe clinical courses of two patients with ISPED in whom reduced-fluence photodynamic therapy (RFPDT) was performed.

Case reports: Two patients (a 38-year-old woman and a 42-year-old man) were diagnosed with ISPED. In both patients, indocyanine green angiography revealed an area of choroidal vascular hyperpermeability including hyperfluorescent PED, which was evident at the subfoveal area. Both patients underwent RFPDT at an energy of $25 \mathrm{~J} / \mathrm{cm}^{2}$. The PED was seen to have resolved 1 month after the treatment and visual acuity was maintained or improved. There was no posttreatment recurrence of PED after 6 months to 1 year, and no treatment-related adverse events were observed.

Conclusion: RFPDT may be an effective and safe method of treatment for ISPED with choroidal vascular hyperpermeability.

Keywords: pigment epithelial detachment, central serous chorioretinopathy, reduced-fluence photodynamic therapy

\section{Introduction}

Pigment epithelial detachment (PED) is a condition in which the retinal pigment epithelium is detached from the Bruch membrane due to the accumulation of fluid or blood between them, which occurs in exudative age-related macular degeneration and other chorioretinal disorders. Idiopathic serous PED (ISPED) is not associated with choroidal neovascularization, meaning that, in most cases, the visual prognosis is good, but patients are aware of distorted vision, and it is known to increase the risk of exudative age-related macular degeneration. ${ }^{1}$ ISPED is categorized into two types, one that exhibits hyperfluorescence under indocyanine green angiography (ICGA), suggesting hyperpermeability of choroidal vessels, and the other that exhibits hypofluorescence. The hyperfluorescent type is extremely similar to the pathology of central serous chorioretinopathy (CSC), and these are regarded as similar disease concepts caused by choroidal hyperpermeability. ${ }^{2,3}$

Very few reports have addressed methods of treatment for ISPED. Standard-fluence photodynamic therapy (PDT) with verteporfin $\left(50 \mathrm{~J} / \mathrm{cm}^{2}\right)$ has been shown to be effective in CSC by improving visual acuity and reducing subretinal fluid. ${ }^{4-6}$ Reduced-fluence PDT (RFPDT) has also been reported as useful in the treatment of CSC. Reibaldi et al evaluated the efficacy of RFPDT compared with standard-fluence PDT for treating chronic CSC, concluding that both PDTs reduce subretinal fluid and improve visual 
acuity in CSC, but RFPDT induces less choriocapillaris damage. ${ }^{7}$ RFPDT (40 J/ $\mathrm{cm}^{2}$ with a shorter exposure time than that of the standard-fluence PDT protocol) has recently been administered to patients with ISPED, and the short-term (3-month) outcomes of this treatment have been reported. ${ }^{8}$

In this study, we report a 6-month to 1-year follow-up of two patients with ISPED in whom ICGA revealed hyperpermeability of the choroidal vessels and ISPED was evident at the subfoveal area, who were treated with RFPDT $\left(25 \mathrm{~J} / \mathrm{cm}^{2}\right.$ with the standard exposure time).

\section{Patients and methods}

This study was approved by the institutional review board of The Jikei University School of Medicine, Tokyo, Japan after fully informed consent had been obtained from both patients. After intravenous administration of the same dose of verteporfin as used in standard-fluence PDT $\left(6 \mathrm{mg} / \mathrm{m}^{2}\right.$ of body surface intravenously for 10 minutes), RFPDT was carried out using the VISULAS 690S PDT system (Carl Zeiss Meditec AG, Jena, Germany) with 83-second exposure, as for standard-fluence PDT, and energy per surface area of $25 \mathrm{~J} /$ $\mathrm{cm}^{2}$. The RFPDT laser spot size was determined by the same method previously reported for CSC; ${ }^{9}$ namely, the spot size was set to cover hyperfluorescent areas (including the whole PED area) identified from mid-phase or late-phase ICGA images. A Cirrus HD-OCT system (Carl Zeiss Meditec) was used for optical coherence tomography (OCT) using protocols of macular cube scan and HD 5-line raster scan. The Heidelberg Retina Angiograph 2 (Heidelberg Engineering $\mathrm{GmbH}$, Heidelberg, Germany) was used for fluorescein angiography (FA) and ICGA.

\section{Results \\ Case I}

The patient was a 38-year-old woman. She had been attending hospital for 15 months, with principal complaints of reduced visual acuity and distorted vision in the right eye. On initial examination, log of the minimum angle of resolution (logMAR) best-corrected visual acuity (BCVA) of the right eye was 0.1 . The images of FA, ICGA, and OCT are described in Figure 1. No serous retinal detachment was evident on OCT images, which showed PED of half the disc diameter in the subfovea, including the fovea, and continuity of the inner and outer segments (IS/OS) line was unclear. FA showed hyperfluorescence in the early phase of contrast enhancement in the same location as the PED, and ICGA revealed hyperfluorescence in the mid-phase and late-phase of contrast enhancement in the same location as the PED, as well as hyperpermeability
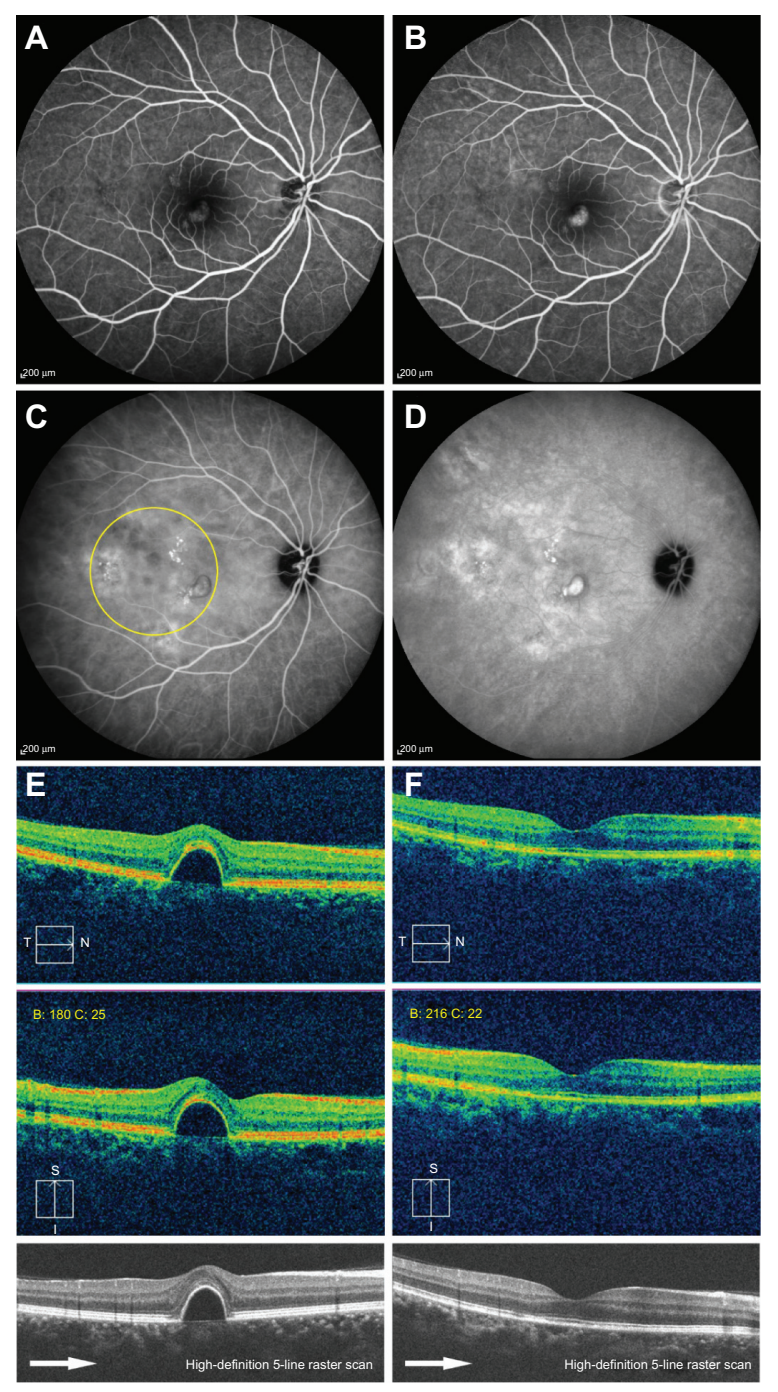

Figure I Fundus and optical coherence tomography findings in the right eye (Case I). Notes: Fluorescein angiograms in the middle (90 seconds after injection) (A) and late (13 minutes after injection) (B) phases, showing leakage in the center of the macula. Indocyanine green angiograms in the middle (6 minutes after injection) (C) and late ( 3 minutes after injection) (D) phases, showing choroidal hyperpermeability in the macular areas. Reduced-fluence photodynamic therapy was performed within the lesion (yellow circle). (E and F) Horizontal and vertical scan images of optical coherence tomography (OCT). Pretreatment OCT reveals pigment epithelial detachment (PED) of half the disc diameter, including the foveal area, and continuity of the inner and outer segments (IS/OS) line in that region is unclear (E). Horizontal and vertical scan images of OCT. One month after treatment, OCT shows that the PED has completely disappeared, and continuity of the IS/OS line has also been restored $(\mathbf{F})$.

of the choroidal vessels in the surrounding area, resulting in a diagnosis of ISPED. In ICGA, no polypoidal lesion or branching vascular network, which is diagnostically seen in polypoidal choroidal vasculopathy, were observed. Because the PED had not improved, RFPDT (spot size: 5,400 $\mu \mathrm{m}$ ) was performed 16 months after onset. The PED was seen to have completely disappeared 1 month after RFPDT. Continuity of the IS/OS line was restored on the OCT images, and logMAR BCVA improved to -0.1 with symptomatic improvement of the 
distorted vision. Visual acuity was maintained at 12 months posttreatment, with no recurrence of PED. No adverse events were seen in this case during follow-up after RFPDT.

\section{Case 2}

The patient was a 42-year-old man. He had been attending hospital for 4 months, with the principal complaint of
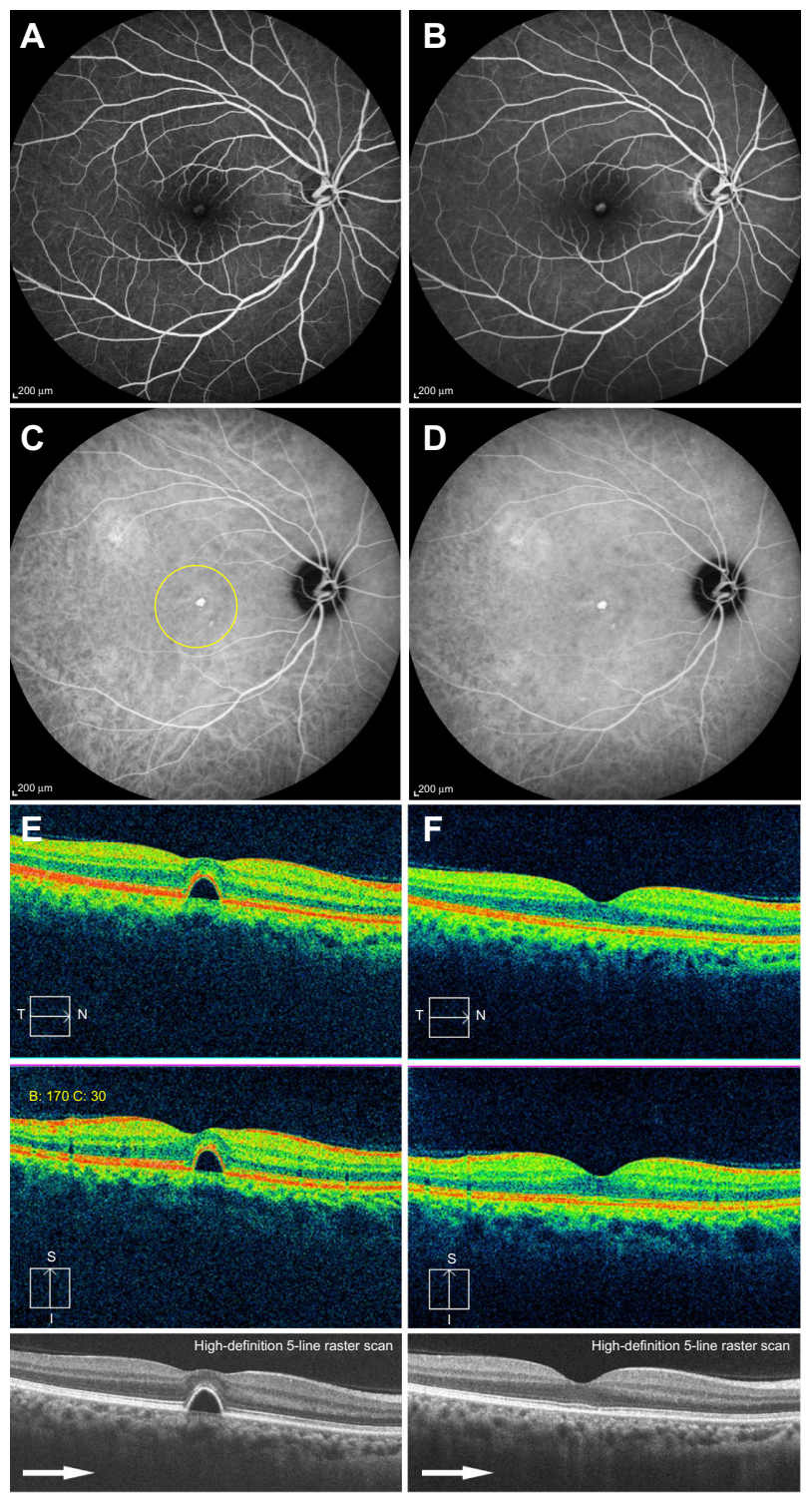

Figure 2 Fundus findings in the right eye (Case 2).

Notes: Fluorescein angiograms in the early (39 seconds after injection) (A) and late (10 minutes after injection) (B) phases, showing leakage in the center of the macula. Indocyanine green angiograms in the middle ( 8 minutes after injection) (C) and late (I2 minutes after injection) (D) phases, showing hyperfluorescence of the pigment epithelial detachment (PED) and weak hyperfluorescence around the PED due to hyperpermeability of the choroidal vessels. Reduced-fluence photodynamic therapy was performed within the lesion (yellow circle). (E and F) Horizontal and vertical scan images of optical coherence tomography (OCT). Pretreatment OCT reveals PED of one-fifth of the disc diameter, including the foveal area, and continuity of the inner and outer segments (IS/OS) line in that area is unclear (E). One month after treatment, OCT shows that the PED has completely disappeared, and continuity of the IS/OS line has also been restored $(\mathbf{F})$. distorted vision in the right eye. On initial examination, $\log$ MAR BCVA of the right eye was -0.2 . The images of FA, ICGA, and OCT were described in Figure 2. No serous retinal detachment was evident on OCT images, which showed serous PED of one-fifth of the disc diameter at the subfovea, and continuity of the IS/OS line was unclear. FA showed hyperfluorescence in the same location as the PED, and ICGA revealed hyperfluorescence of the PED from the mid-phase to the late-phase and weak hyperfluorescence around the PED due to hyperpermeability of the choroidal vessels, resulting in a diagnosis of ISPED. Because the PED had not improved, RFPDT (spot size: 3,300 $\mu \mathrm{m}$ ) was performed 6 months after onset. The PED was seen to have completely disappeared 1 month after RFPDT. Continuity of the IS/OS line was restored on OCT images, and logMAR BCVA was maintained, with symptomatic improvement of distorted vision. Visual acuity was maintained at 6 months posttreatment, with no recurrence of PED. No adverse events were seen in this case during follow-up after RFPDT.

\section{Discussion}

This report presents the good therapeutic course of two patients with ISPED with choroidal hyperpermeability, who were treated with RFPDT. Although Case 1 had suffered from this disorder for over 1 year, the PED was seen to have completely disappeared 1 month after treatment, and this good condition was maintained at 1 year following the procedure without the need for additional treatment. In Case 2, the PED was also seen to have completely disappeared 1 month after treatment.

Both patients exhibited hyperfluorescence on ICGA that was suggestive of choroidal hyperpermeability, and although serous retinal detachment was not present, their pathology closely resembled that of CSC. With respect to the natural course of PED associated with CSC, Mudvari et al report that, during a mean follow-up period of 49 months, it disappeared spontaneously in $65 \%$ of cases, and mean visual acuity improved. ${ }^{10}$ However, they also report that visual acuity declined in $50 \%$ of their patients in whom PED was present at the subfovea, ${ }^{10}$ and this may correspond to our patients, whose visual acuity could be expected to decline in future.

Very few reports have addressed methods of treatment for ISPED. Goto et al performed RFPDT to treat PED (at the subfoveal area) with hyperpermeability of the choroidal vessels, and they report the short-term (3-month) outcomes of this treatment. ${ }^{8}$ They mention that the PED had completely disappeared 1 month after RFPDT in 93\% of cases, visual acuity improved significantly during 3 months' posttreatment 
follow-up, and no recurrence was observed. ${ }^{8}$ For their RFPDT procedure, they chose to reduce the fluence to $40 \mathrm{~J} / \mathrm{cm}^{2}$ by using a shorter exposure time than that of the standard-fluence PDT protocol. ${ }^{8}$ We chose to perform RFPDT at an even lower fluence of $25 \mathrm{~J} / \mathrm{cm}^{2}$, and we obtained similarly good results. The follow-up period after RFPDT treatment reported by Goto et $\mathrm{al}^{8}$ is short (only 3 months), but the two patients in our study were followed for 6 months to 1 year, and no recurrence of PED was observed. Reducing PDT energy is believed to decrease the incidence of persistent choriocapillaris hypoperfusion, pigmentary changes of retinal pigment epithelium, choroidal atrophy, and secondary choroidal neovascularization as PDT-induced adverse events, ${ }^{7,9}$ and, if an equivalent therapeutic effect can be obtained, the lowerfluence PDT used in our method of treatment may make it superior to that reported by Goto et al. ${ }^{8}$

The main limitation of this study was that it was shortterm and retrospective with a small number of cases. To establish the most effective and safest PDT protocol for treating ISPED, further studies are required that investigate long-term courses of treatment in larger numbers of patients compared with controls (untreated cases).

\section{Conclusion}

Excellent therapeutic results were obtained in two patients with ISPED in whom ICGA revealed choroidal hyperpermeability, who were treated with RFPDT at a reduced fluence of $25 \mathrm{~J} / \mathrm{cm}^{2}$.

\section{Acknowledgment}

This study was supported by a grant from the Vehicle Racing Commemorative Foundation (TH and HT).

\section{Disclosure}

The authors report no conflicts of interest in this work.

\section{References}

1. Meredith TA, Braley RE, Aaberg TM. Natural history of serous detachments of the retinal pigment epithelium. Am J Ophthalmol. 1979;88(4): 643-651.

2. Lewis ML. Idiopathic serous detachment of the retinal pigment epithelium. Arch Ophthalmol. 1978;96(4):620-624.

3. Giovannini A, Scassellati-Sforzolini B, D'Altobrando E, Mariotti C, Rutili T, Tittarelli R. Choroidal findings in the course of idiopathic serous pigment epithelium detachment detected by indocyanine green videoangiography. Retina. 1997;17(4):286-293.

4. Chan WM, Lam DS, Lai TY, Tam BS, Liu DT, Chan CK. Choroidal vascular remodelling in central serous chorioretinopathy after indocyanine green guided photodynamic therapy with verteporfin: a novel treatment at the primary disease level. Br J Ophthalmol. 2003;87(12): 1453-1458.

5. Yannuzzi LA, Slakter JS, Gross NE, et al. Indocyanine green angiography-guided photodynamic therapy for treatment of chronic central serous chorioretinopathy: a pilot study. Retina. 2003;23(3): 288-298.

6. Cardillo Piccolino F, Eandi CM, Ventre L, Rigault de la Longrais RC, Grignolo FM. Photodynamic therapy for chronic central serous chorioretinopathy. Retina. 2003;23(6):752-763.

7. Reibaldi M, Cardascia N, Longo A, et al. Standard-fluence versus low-fluence photodynamic therapy in chronic central serous chorioretinopathy: a nonrandomized clinical trial. Am J Ophthalmol. 2010;149(2):307-315. e2

8. Goto S, Gomi F, Ueno C, Nishida K. Reduced-fluence photodynamic therapy for subfoveal serous pigment epithelial detachment with choroidal vascular hyperpermeability. Am J Ophthalmol. 2012;154(5): 865-871. e1.

9. Ohkuma Y, Hayashi T, Sakai T, Watanabe A, Tsuneoka H. One-year results of reduced fluence photodynamic therapy for central serous chorioretinopathy: the outer nuclear layer thickness is associated with visual prognosis. Graefes Arch Clin Exp Ophthalmol. 2013;251(8): 1909-1917.

10. Mudvari SS, Goff MJ, Fu AD, et al. The natural history of pigment epithelial detachment associated with central serous chorioretinopathy. Retina. 2007;27(9):1168-1173.
Clinical Ophthalmology

\section{Publish your work in this journal}

Clinical Ophthalmology is an international, peer-reviewed journal covering all subspecialties within ophthalmology. Key topics include: Optometry; Visual science; Pharmacology and drug therapy in eye diseases; Basic Sciences; Primary and Secondary eye care; Patient Safety and Quality of Care Improvements. This journal is indexed on Submit your manuscript here: http://www.dovepress.com/clinical-ophthalmology-journal

\section{Dovepress}

PubMed Central and CAS, and is the official journal of The Society of Clinical Ophthalmology (SCO). The manuscript management system is completely online and includes a very quick and fair peer-review system, which is all easy to use. Visit http://www.dovepress.com/ testimonials.php to read real quotes from published authors. 